\title{
Measuring Gas Concentration and Wind Intensity in a Turbulent Wind Tunnel with a Mobile Robot
}

\author{
Dani Martínez, ${ }^{1}$ Javier Moreno, ${ }^{1}$ Marcel Tresanchez, ${ }^{1}$ \\ Eduard Clotet, ${ }^{1}$ Juan Manuel Jiménez-Soto, ${ }^{2}$ Rudys Magrans, ${ }^{1}$ \\ Antonio Pardo, ${ }^{3}$ Santiago Marco, ${ }^{2,3}$ and Jordi Palacín ${ }^{1}$ \\ ${ }^{1}$ Department of Computer Science and Industrial Engineering, University of Lleida, Jaume II 69, 25001 Lleida, Spain \\ ${ }^{2}$ Signal and Information Processing for Sensing Systems, Institute for Bioengineering of Catalonia, \\ Baldiri Reixac 10-12, 08028 Barcelona, Spain \\ ${ }^{3}$ Department of Electronics, University of Barcelona, Martí i Franquès 1, 08028 Barcelona, Spain
}

Correspondence should be addressed to Jordi Palacín; palacin@diei.udl.cat

Received 12 November 2015; Revised 24 December 2015; Accepted 3 January 2016

Academic Editor: Kewei Zhang

Copyright (C) 2016 Dani Martínez et al. This is an open access article distributed under the Creative Commons Attribution License, which permits unrestricted use, distribution, and reproduction in any medium, provided the original work is properly cited.

\begin{abstract}
This paper presents the measurement of gas concentration and wind intensity performed with a mobile robot in a custom turbulent wind tunnel designed for experimentation with customizable wind and gas leak sources. This paper presents the representation in different information layers of the measurements obtained in the turbulent wind tunnel under different controlled environmental conditions in order to describe the plume of the gas and wind intensities inside the experimentation chamber. The information layers have been generated from the measurements gathered by individual onboard gas and wind sensors carried out by an autonomous mobile robot. On the one hand, the assumption was that the size and cost of these specialized sensors do not allow the creation of a net of sensors or other measurement alternatives based on the simultaneous use of several sensors, and on the other hand, the assumption is that the information layers created will have application on the development and test of automatic gas source location procedures based on reactive or nonreactive algorithms.
\end{abstract}

\section{Introduction}

Robotics has become an excellent alternative for developing measurement platforms in applications requiring mobility and the possibility of implementing a specific behavior or path planning procedures. Many robot algorithms, procedures, and mechanics which have been widely used in robotics are inspired and based on nature agents to take advantage of thousands of evolution years [1]. Such bioinspired influences are often present on robotic shaping, physics, and robot intelligence which can be designed based on animal strategies and other behaviors in order to incorporate their heuristics and senses. However, most of the robots are designed and optimized in order to be fulfilled with a specific behavior or application, so they used to be configured to have a restricted number of implemented functions and sensors [2]. Examples of such bioinspired sensors are the chemical sensors which can imitate the sense of olfaction in the development of many automatic applications [3] such as odor classification [4]. In recent years different sensor mechanisms have been used with robots in order to imitate the human odor sensing capability [5]. For example, in [6] a mobile robot with wind, gas, and temperature sensors was used to define an ambient intelligence application in order to increase human comfort. Mobile gas sensors can be applied to detect the location of illegal chemicals and volatile substances from explosives [7] and to supervise gas concentration on industries or laboratories in order to ensure a human-safe workplace [8].

However, the variability of environment agents such as wind conditions, freed gas agent, gas injection rate, and chemical sensor used is prone to the development of automatic gas leak source detection. Such variability has been analyzed by modeling the dynamics of the environments by 
means of the development of gas distribution maps [9] which have been enhanced based on the accurate positioning of the gas concentration measurements by means of a Simultaneous Location And Mapping (SLAM) method [10]. For example, in [11] the overall performance of a Fast-SLAM algorithm for autonomous robot self-location and navigation has been improved by means of a distributed structure and in [12] the SLAM method has been applied based on the information provided by a single monocular camera.

The information maps are proposed to model the gas diffusion behavior inside a delimited area and can have direct application in the development of automatic gas sourcefinding applications by a mobile robot. For example, [13] proposes the creation of gas concentration grid maps with a mobile robot by convolving robot locations and sensors reading with a radially symmetric two-dimensional Gaussian diffusion function although this proposal cannot be applied in this paper because of the strong unidirectional airflow used in the wind tunnel. The same procedure was also applied in [14] in order to identify multiple odour sources, using gas sensors which are not capable of gathering gas concentrations levels directly. Similarly, in [15] different distribution maps have been used to discriminate the location of different gas sources and in [16] a multirobot system was proposed in order to obtain gas distribution maps and develop experiments with different gas source-finding algorithms. In a similar direction, [17] proposes a methodology to generate large gas distribution maps, but in this case, by using a flying drone which also includes onboard sensors in order to measure gas concentration and wind intensity and direction. Finally, [18] presents a dataset of different chemical compounds obtained under different environmental conditions by defining a set of experiments carried out in a turbulent wind tunnel. In this case, the measurement of the gas concentration was performed with an array of sensors placed along the experimentation chamber.

The new contribution of this paper is the measurement and representation of gas concentration and wind intensity in a turbulent wind tunnel in different operational conditions by using a mobile robot. This paper details the assessment of different experimental setups performed with a mobile robot equipped with a gas and wind sensor and the creation of different maps or information layers by referencing, positioning, grouping, and processing the data gathered by the mobile sensors. All the experiments described in this paper have been carried out in a customized turbulent wind tunnel with different gas freeing mechanisms and localization. The graphical results obtained, interpreted as information layers, describes wind intensity and gas concentration parameters whose different tendencies are presented and discussed.

\section{Materials and Methods}

The materials used in this paper are the components used in the implementation of the turbulent wind tunnel and the mobile robot used to perform mobile gas and wind intensity measurements. Additionally, the methods used are the procedures applied to obtain the different information layers that describe the wind intensity and gas concentration parameters inside the turbulent wind tunnel.

2.1. Turbulent Wind Tunnel. The experimental tests developed in this paper have been carried out in a customized box-shaped wind tunnel. This construction was made from aluminum profiles and Styrodur ${ }^{\circledR}$ insulation boards with the following inner dimensions: $3.5 \mathrm{~m}$ (width) $\times 4.8 \mathrm{~m}$ (large) $\times$ $1.8 \mathrm{~m}$ (height). The wind tunnel has 4 extractor fans installed: one placed in the center of the front wall, pushing air inside the chamber, and the other three placed at the same height at the back wall, extracting air outside. This configuration has been selected in order to facilitate the turbulent diffusion of the gas while generating a central wind plume profile. The model of the extractor fans is HXM-400 by Soler \& Palau, and the power applied to the fans can be adjusted in order to simulate different ambient situations. This custom built structure is placed in a laboratory with two practicable large windows ( $8 \mathrm{~m}$ wide) that are managed to assure the adequate renovation of the air inside the chamber by avoiding polluted air recirculation. Figure 1(a) shows a photo of the wind tunnel and the three fans. The tunnel has three predefined different possible emplacements for the gas freeing mechanism against the frontal wall. The gas source consisted in a small pipe with a pullout $8 \mathrm{~cm}$ fan on the top that evaporates pure acetone from a small glass at the base of the pipe (Figure 1(b)). This mechanism is placed at $40 \mathrm{~cm}$ height and evaporates acetone at an average constant rate of $32 \mathrm{~mL} / \mathrm{h}$.

2.2. Mobile Robot. The main operational platform for this work is a custom mobile robot (Figure 2) [6]. The structure of the mobile robot is composed by aluminum profiles, plastic covers, and other ABS 3D printed pieces. The motion of the mobile robot is handled by two DC motors (P205 Micro Motors s.r.l.) connected to a motor control board (Cortex 4 ARM microcontroller). A personal computer (dual-core $1.6 \mathrm{GHz}, 4 \mathrm{~GB}$ RAM, and $128 \mathrm{~GB}$ SSD) with Windows 8.1 operates as the central processing unit and manages the access to all sensors and actuators by using USB connections. The navigation and obstacle detection procedures obtain environmental information provided by a Hokuyo UTM30LX. This laser sensor gets 1,081 points in polar form from a $2 \mathrm{D}$ angular range of $-135^{\circ}$ to $+135^{\circ}$ and a maximum linear range of $30 \mathrm{~m}$.

The mobile robot includes a ppbRAE 3000 Photoionization Detector (PID) by RAE Systems attached to its structure and placed at $45 \mathrm{~cm}$ from the floor, at approximately the horizontal plane defined by the gas source used in the experiments. The PID measures gas intensity in parts per billion (ppb), has an effective maximum sampling rate of $1 \mathrm{~Hz}$, and is configured to measure acetone and diethyl volatile substances. In addition, the robot includes a Windsonic anemometer (Gill Instruments Inc.) in order to measure the wind speed and its direction. The robot control routines and procedures are implemented by using MATLAB script functions although some heavy-computational procedures such as the Fast-SLAM algorithm have been implemented in $C$ language and used as fast MATLAB MEX functions which 


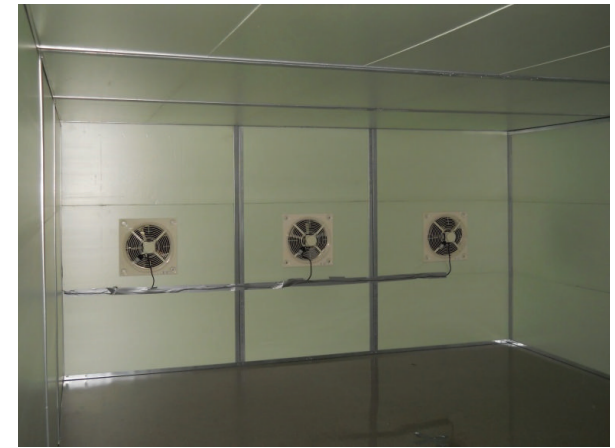

(a)

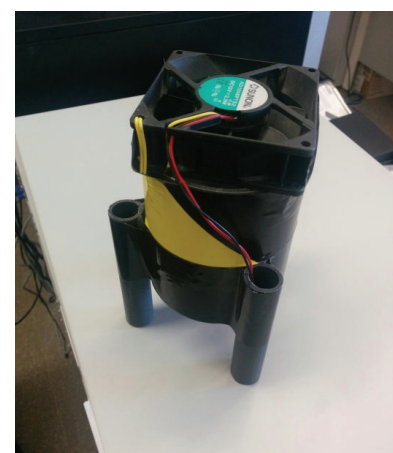

(b)

FIGURE 1: (a) Interior of the custom wind tunnel and (b) detail of the gas source evaporator pipe.

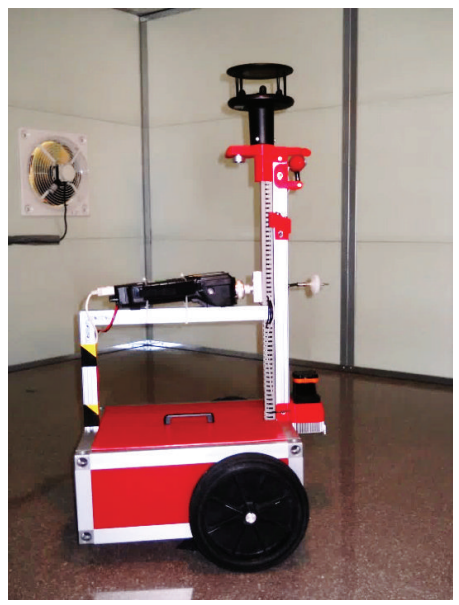

Figure 2: Mobile robot used in the measurements.

provides a mobile robot position estimate in less than one second and additional time to acquire information from the sensors at a sampling rate of $1 \mathrm{~Hz}$.

2.3. Information Layers. The interpretation as information layers of the data measured by the onboard sensors of the mobile robot requires the application of a self-localization procedure based on the SLAM methodology in order to estimate the absolute position of the mobile robot in the wind tunnel. The data gathered by the laser range sensor are processed as binary image by applying an occupancy grid SLAM methodology $[10,19]$ combined with an image template matching tracking method [20]. This process compares the actual set of scanned points with the points obtained from the next laser scan in order to estimate mobile robot displacement. The mobile robot pose is returned as the absolute $x$ axes and $y$-axes position as well as the orientation of the robot in degrees. In order to speed up the SLAM process the search window of the template matching implementation has been limited according the estimate mobile robot displacement. The central process unit of the mobile robot is able to perform navigation and onboard sensor sampling in real-time. The raw information of all the onboard sensors can be stored

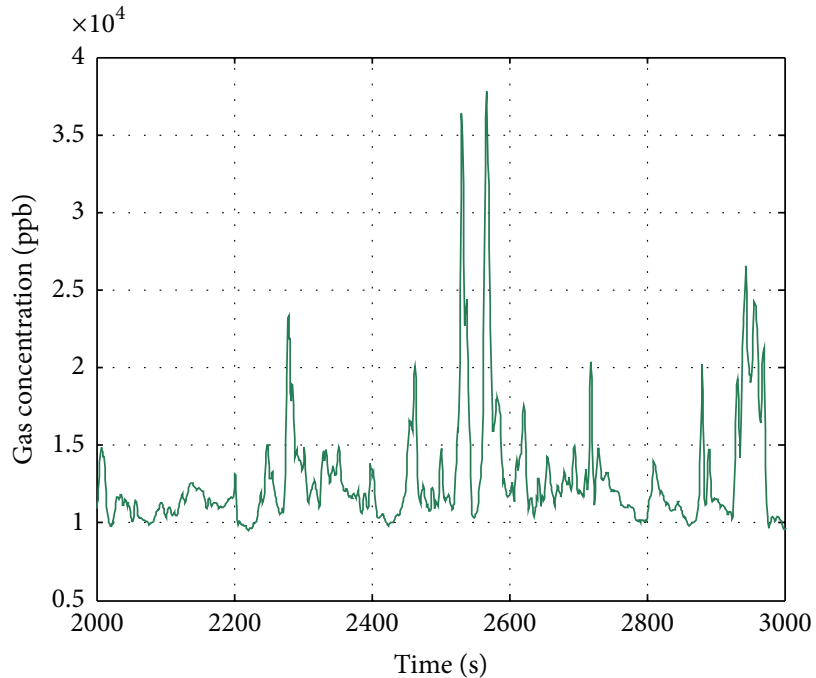

FIGURE 3: Typical evolution of gas concentration samples during a mobile robot exploration.

in a log register for additional verification or further offline analysis. As an example, Figure 3 shows typical raw gas concentration measurements obtained with the PID while the robot is exploring the wind tunnel. Figure 3 shows the presence of some typical concentration peaks whose height depends on the airflow turbulences and on the proximity to the gas source. In practice, the mobile robot can detect gas concentration peaks of different amplitudes when passing several times around an absolute $(x, y)$ location inside the wind tunnel. In general, the gas concentration peaks tend to be representative of the gas concentration in the turbulent airflow when grouping sensory information in small region areas so all the information gathered with the PID and the wind intensity sensors has been grouped in small representative cells that conform grid maps or information layers in order to enhance the overall interpretation of the data gathered with the onboard sensors of the mobile robot.

The procedure used to generate the information layers with the gas and wind information has different steps. In a preliminary step, the mobile robot creates a detailed map 
of the experimentation area by applying a complete SLAM procedure during a simple exploration. This map never changes during the experiments so it is saved as a reference map in order to speed up the computations performed during an exploration by avoiding continuous map update (applying only a map-based self-location procedure).

As a main experimental step, the mobile robot performs a semirandom exploration (Figure 4(a)) during 1 hour inside the wind tunnel in order to obtain the different information layers. In this paper, the path planning of the mobile robot leads the robot straight (at a maximum velocity of $0.2 \mathrm{~m} / \mathrm{s}$ ) until detecting an obstacle and then turning a random number of degrees to its left and repeating the procedure. This exploration assures complete coverage of the wind tunnel. The onboard PID and anemometer sensors are sampled at $1 \mathrm{~Hz}$ in order to obtain synchronized environmental measurements which are used by the mobile robot to generate different information layers (Figure 4(b)). The parameters used in this proposal are the gas concentration (ppb) and the absolute wind speed $(\mathrm{m} / \mathrm{s})$. In this paper, the maximum velocity of the mobile robot is lower than the background wind intensity measured when the mobile robot is stopped so the absolute wind speed obtained is not corrected with the velocity information of the mobile robot. Figure 4 includes large arrows which illustrate the location of the fans and the direction of the wind generated which will be the same for all analysis performed in this paper.

Figure 4(b) gives a relative representation of the size of the square boxes or cells used to group the information of the sensors (in cells of $0.2 \times 0.2$ meters). The cells are filled with the data obtained during the exploration and continuously analyzed in order to show their local maximum, average and standard deviation values. All gathered sensor data have the same weight while the random path assures the same coverage and thus the same number of pieces of information in all the cells. As an example, Figure 4(b) includes the information layers with the maximum gas intensity measured in the boxes which are filled with colors for easier interpretation. In this case the gas source was on the left of the frontal wall. The low-resolution information layer can be smoothed (Figure 4(c)) for an improved visualization, in this case, by applying a biharmonic spline interpolation [21] which has been widely used on 2D geophysical mapping since the 1970s [22]. As an example, Figure 4(c) shows a smoothed highresolution interpretation of the information layer (1 square area is smoothed as $8 \times 8$ pixels) corresponding to the gas distribution previously shown in Figure 4(b) and, similarly, Figure 4(d) shows a smoothed high-resolution interpretation of the wind information layer.

\section{Measuring inside the Turbulent Wind Tunnel}

This section presents the measurement results obtained by means of different experiments carried out with a gas source placed at different locations and with different settings on wind intensity. The hypothesis is that the information layers showing the gas concentration and wind intensity describe consistent background information which will have application in the tests of different gas source-finding algorithms.

3.1. Wind Profile. This section presents the experiments performed in order to obtain the information layers which describes the wind profile inside the wind tunnel when the mobile robot performs a semirandom path-planning exploration during 1 hour. In these experiments, the evaporation of the gas is started when starting the experiment. Figure 5 shows the smoothed information layers showing the maximum, average, and standard deviation value of the wind intensity measured. These information layers have been obtained in three experiments with $33 \%, 66 \%$, and $100 \%$ power applied to the fans; similar information layers have been obtained when repeating the procedure of the experiments.

In the case with the power applied to the fans set to $33 \%$ (Figure 5), the plume originated by the main pullout fan is clearly defined in the information layer showing the maximum values of the peaks, the average values, and the standard deviation of the wind intensity. The maximum peak of the wind speed measured was $2.37 \mathrm{~m} / \mathrm{s}$ just in front of the pullout fan and this value decreased to $0.51 \mathrm{~m} / \mathrm{s}$ outside the wind plume. The average wind velocity value in front of the pullout fan was $1.59 \mathrm{~m} / \mathrm{s}$ and this value decreased to $0.28 \mathrm{~m} / \mathrm{s}$ outside the plume. The standard deviation of the wind velocity reached values up to $0.59 \mathrm{~m} / \mathrm{s}$ just in front of the pullout fan and decreased to $0.12 \mathrm{~m} / \mathrm{s}$ outside the plume.

In the case with the power applied to the fans set to $66 \%$ (Figure 5), the plume originated by the main pullout fan is again clearly defined in the information layer. The maximum peak of the wind speed measured was $3.63 \mathrm{~m} / \mathrm{s}$ just in front of the pullout fan and this value decreased to $0.70 \mathrm{~m} / \mathrm{s}$ outside the wind plume. The average wind velocity value in front of the pullout fan was $2.49 \mathrm{~m} / \mathrm{s}$ and this value decreased to $0.39 \mathrm{~m} / \mathrm{s}$ outside the plume. The standard deviation of the wind velocity has a maximum deviation of $1.16 \mathrm{~m} / \mathrm{s}$ just in front of the pullout fan and decreased to $0.16 \mathrm{~m} / \mathrm{s}$ outside the plume.

In the case with the power applied to the fans set to $100 \%$ (Figure 5), the plume originated by the main pullout fan also appears to be clearly defined in the information layer. The maximum peak of the wind speed measured was $5.03 \mathrm{~m} / \mathrm{s}$ just in front of the pullout fan and this value decreased to $0.91 \mathrm{~m} / \mathrm{s}$ outside plume. The average wind velocity value in front of the pullout fan was $4.02 \mathrm{~m} / \mathrm{s}$ and this value decreased to $0.52 \mathrm{~m} / \mathrm{s}$ outside the plume. The standard deviation of the wind velocity reached a maximum deviation of $3.09 \mathrm{~m} / \mathrm{s}$ in front of the pullout fan and decreased to $0.20 \mathrm{~m} / \mathrm{s}$ outside the plume.

In general, the information layers of Figure 5 show a central airflow plume going from the pushing fan to the extractor fans. The shape of the diffusion plume is particularly noticeable on the maximum and on the average wind information layers. Results show that the size of the plume observed in the information map of the standard deviation (gas intensity variations) decreases as the wind intensity (or power fan) increases. 


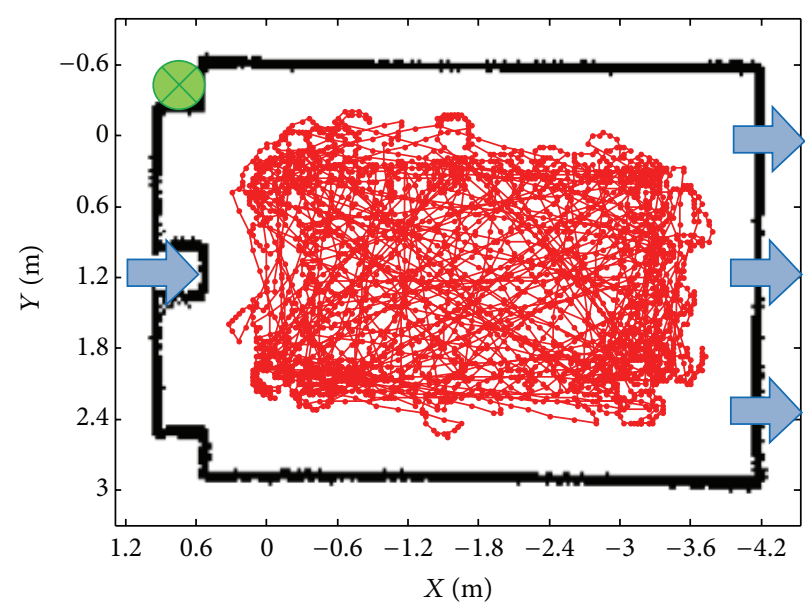

(a)

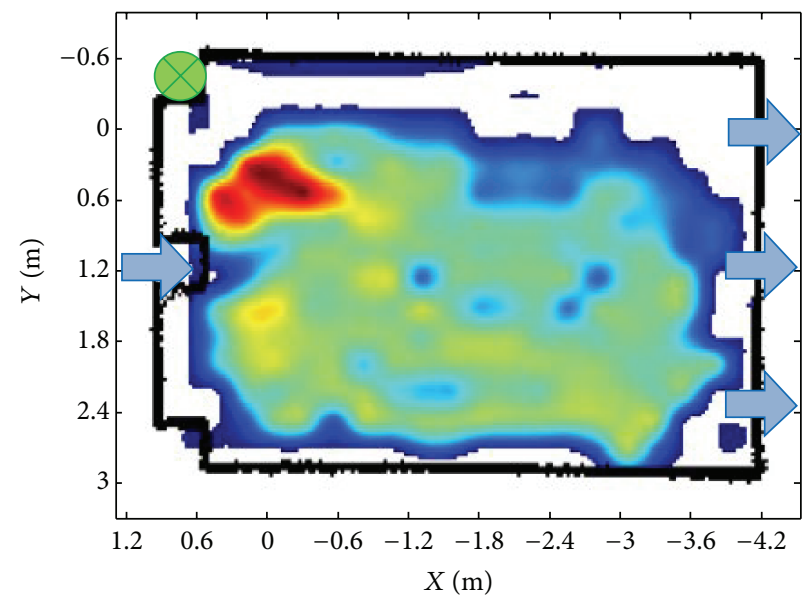

(c)

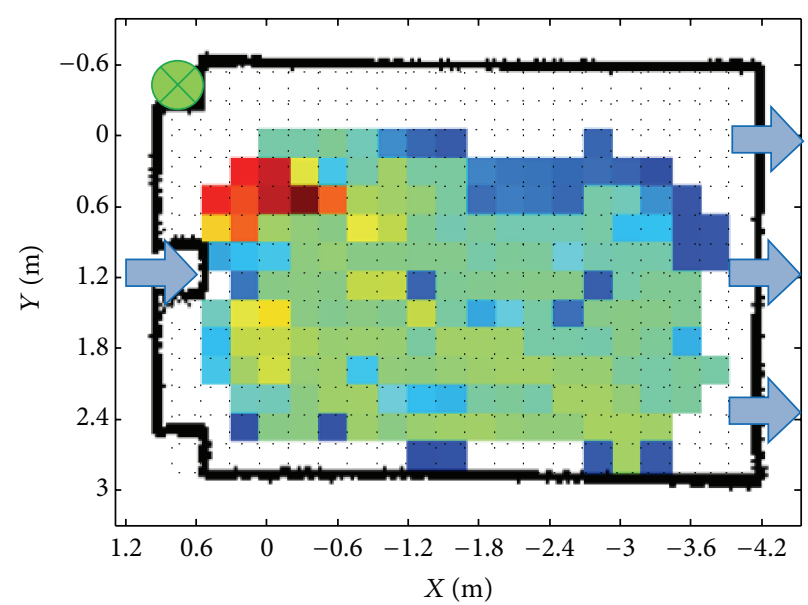

(b)

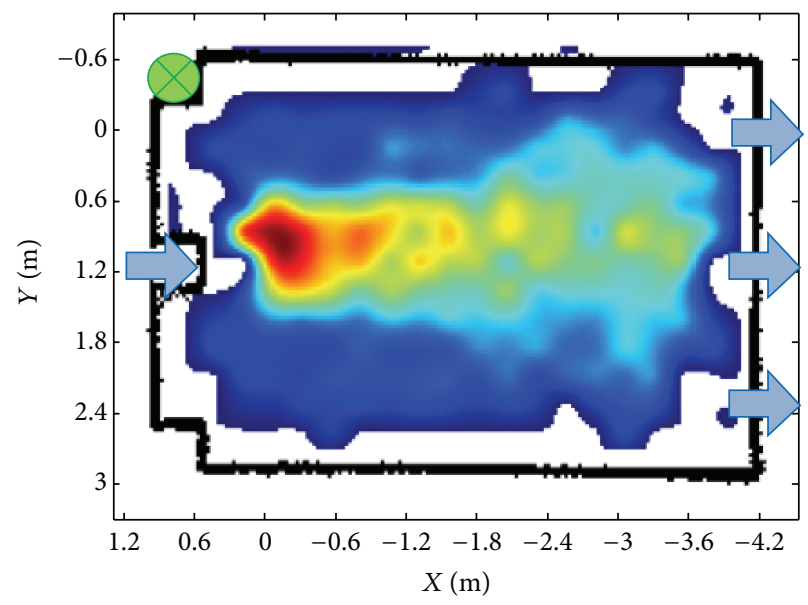

(d)

FIGURE 4: (a) Robot semirandom exploration path (red line): the blue arrows depict the location and direction of the fans and the green circle depicts the location of the gas source. (b) Information layer showing the maximum gas intensity concentration measured. (c) Smoothed information layer showing of the maximum gas concentration measured. (d) Smoothed information layer showing the maximum wind intensity measured by the mobile robot.

3.2. Gas Distribution Profile. This section presents the experiments performed in order to obtain the information layers which describe the gas diffusion profile inside the wind tunnel when the mobile robot performs semirandom pathplanning exploration during 1 hour. The minimum time-lapse between experiments was 6 hours and a gas concentration baseline of $0 \mathrm{ppb}$ was verified before initiating an experiment. Figure 6 shows the smoothed information layers showing the maximum gas peak intensity measured, average concentration, and standard deviation of the gas concentration measured. These information layers have been obtained with different experimental setups labeled as cases and also include the description of the fan power applied in parenthesis. Finally, similar information layers have been obtained when repeating the procedure of the experiments.

Cases 1 and 2 of Figure 6 show the information layers obtained when a single gas source (green circle with a cross) was placed at the corner of the wind tunnel under different wind intensities. Case 3 defines an experiment with the gas source centered in front of the pushing fan. Finally, Case 4 defines an experiment with two gas sources placed on opposite corners of the frontal wall. Figure 6 presents the information layers obtained showing the maximum gas intensity (peak) measured and the average and standard deviation of these values of gas intensity.

Case 1 of Figure 6 shows the information layers of the gas distribution with all fans powered at 33\% and with the gas source located on the left of the frontal wall (Figure 6, green circle). The maximum value of the gas concentration was $38 \mathrm{ppm}$ at a location very close to the gas source and with a large lateral plume, outside this plume the maximum value of the gas concentration decreased to an average value of $13 \mathrm{ppm}$. The average value (Figure 6, Case 1) of the gas concentration had a sharp peak of $34 \mathrm{ppm}$ at a location very close to the gas source and outside this peak the average intensity decreased to $9 \mathrm{ppm}$. The standard deviation (Figure 6, Case 1) showed a narrow peak with a value of $17 \mathrm{ppm}$ and values of $1.8 \mathrm{ppm}$ outside this peak. Therefore, 
Fans

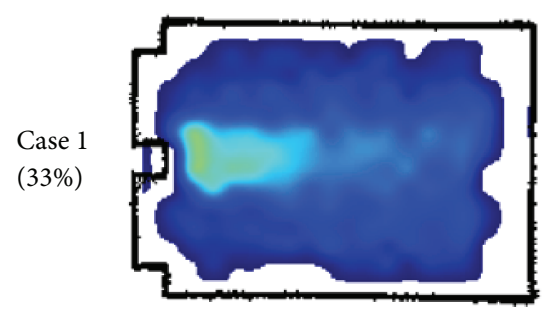

$\operatorname{Max}=2.4 \mathrm{~m} / \mathrm{s}$

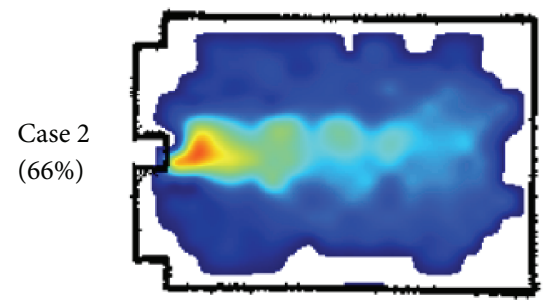

$\operatorname{Max}=3.6 \mathrm{~m} / \mathrm{s}$

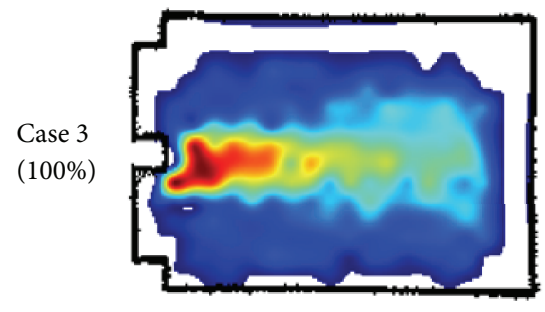

$\operatorname{Max}=5 \mathrm{~m} / \mathrm{s}$

$(\mathrm{m} / \mathrm{s})$

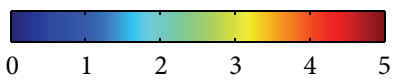

Wind (average)

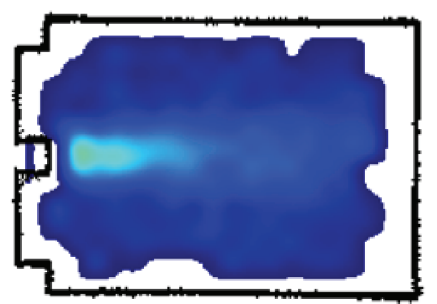

$\operatorname{Max}=1.6 \mathrm{~m} / \mathrm{s}$

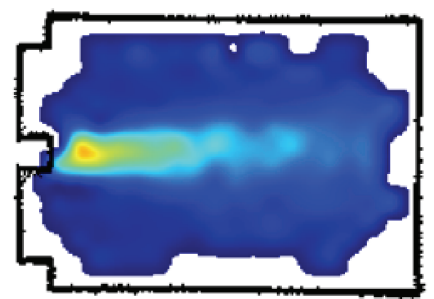

$\operatorname{Max}=2.5 \mathrm{~m} / \mathrm{s}$

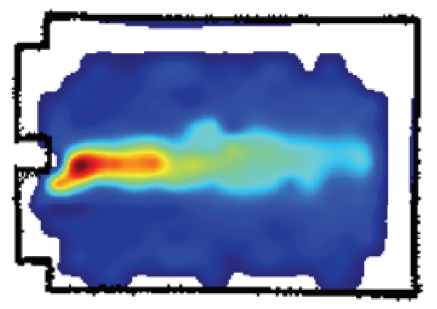

$\operatorname{Max}=4 \mathrm{~m} / \mathrm{s}$

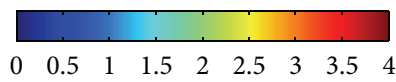

Wind (standard deviation)

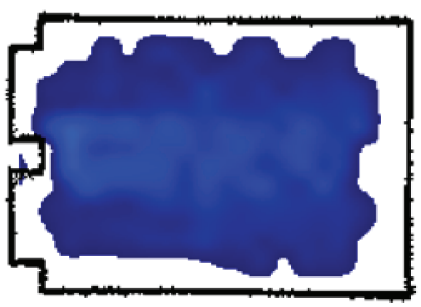

$\operatorname{Max}=0.6 \mathrm{~m} / \mathrm{s}$

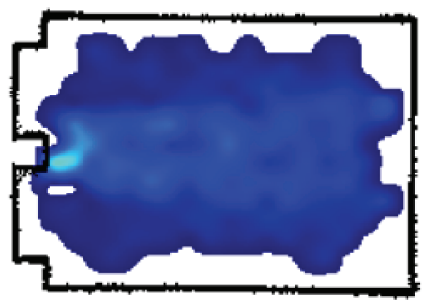

$\operatorname{Max}=1.2 \mathrm{~m} / \mathrm{s}$

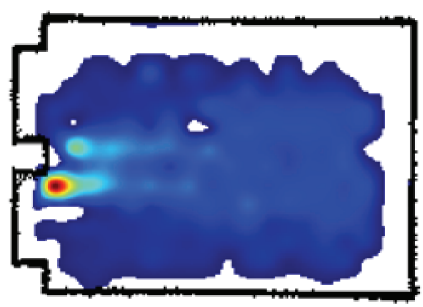

$\operatorname{Max}=3.1 \mathrm{~m} / \mathrm{s}$

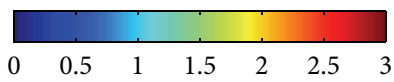

FIGURE 5: Information layers of the wind intensity in different experimental cases.

according the information layers obtained from Case 1 experiment, the largest gas plume is obtained when registering the gas intensity peaks measured by the mobile robot and then this information layer is adequate in order to propose exploratory trajectories in a typical gas source localization application. Additionally, the information layer showing the standard deviation of the gas concentration measurements can be used to directly define stop criteria during a gas location experiment because the standard deviation of the measurements near the gas source used to be from 2 to 8 times of the background standard deviation level.

Case 2 of Figure 6 shows the information layers corresponding to the gas concentration measured with all fans powered at $66 \%$ and with the gas source located on the left of the frontal wall. The maximum gas concentration shown in Figure 6, Case 2, was located near the gas source with a maximum intensity of $49 \mathrm{ppm}$ but this peak was very narrow and the maximum intensity of the peaks measured decreased to $11 \mathrm{ppm}$ in a very short distance. In Figure 6, Case 2, the information layer showing the average values of the gas concentration measured showed peak intensity close to the gas source with values of $21 \mathrm{ppm}$ and a very constant background level of 9 ppm around all the area of the wind tunnel. The information layer corresponding to the standard deviation (Figure 6, Case 2) was very similar to the previous case with a narrow peak with a value of $9 \mathrm{ppm}$ and values of 1.2 ppm outside this peak. Therefore, in Figure 6, Case 2, the gas diffusion plume is not clearly defined in any information layer so it is expected that a mobile robot will experience problems if trying to locate a gas source based only on the gas concentration measured by the onboard sensor.

In Figure 6, Case 3, the gas source was placed in front of the pullout fan and directly exposed to the main airflow plume and the fans were set to $33 \%$ of its maximum power. Results from the maximum gas concentration (Figure 6, Case 3) show clearly a gas plume with a maximum gaspeak concentration of $9 \mathrm{ppm}$ and with $5 \mathrm{ppm}$ outside the plume. The average information layer (Figure 6, Case 3) has a maximum value just in front the propeller fan with $7 \mathrm{ppm}$ but similar larger values were also found next to the surrounding walls with an almost uniform value of $5 \mathrm{ppm}$ along all the inner area of the wind tunnel. In this case (Figure 6, Case 3), the standard deviation reaches its maximum value, $3 \mathrm{ppm}$ at the exit wall, and the deviation in the inner surface is around $0.8 \mathrm{ppm}$. Therefore, the expectation is that a mobile robot will be able to locate the gas source by following the gas plume described in the information layer showing gas peak concentration values. 
Case

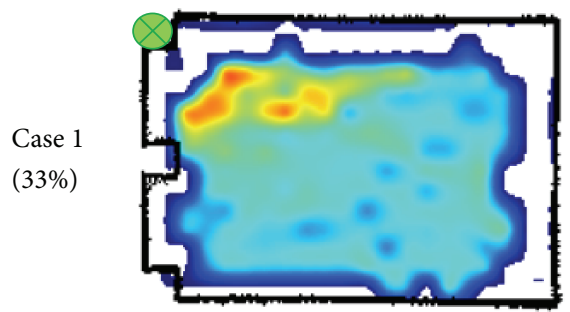

$\operatorname{Max}=38 \mathrm{ppm}$

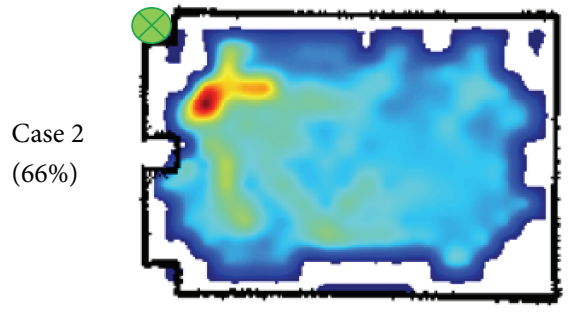

$\operatorname{Max}=49 \mathrm{ppm}$

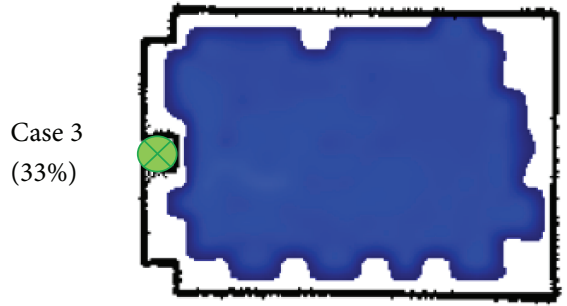

$\operatorname{Max}=9 \mathrm{ppm}$

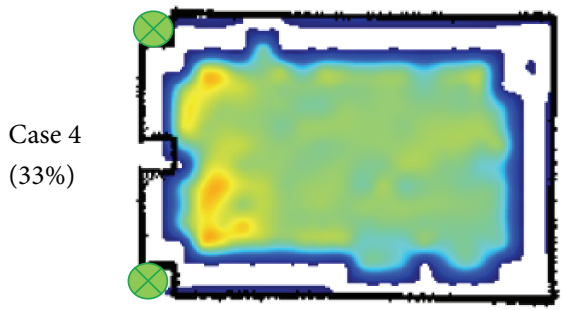

$\operatorname{Max}=34.5 \mathrm{ppm}$

(ppm)

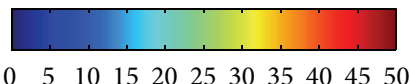

Gas (average)

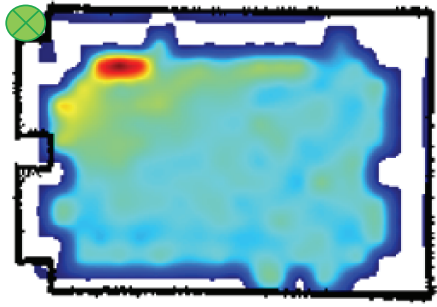

$\operatorname{Max}=34 \mathrm{ppm}$

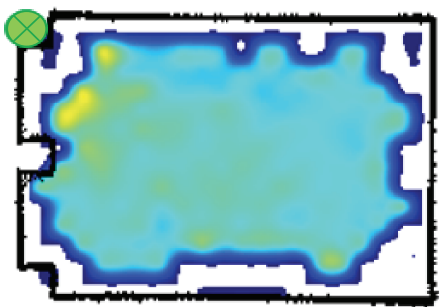

$\operatorname{Max}=21 \mathrm{ppm}$

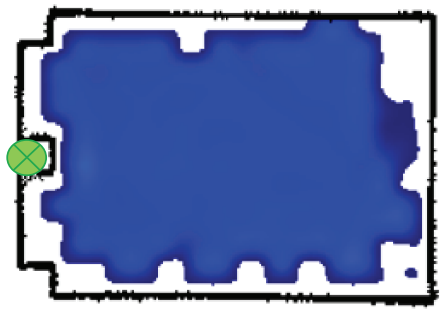

$\operatorname{Max}=7 \mathrm{ppm}$

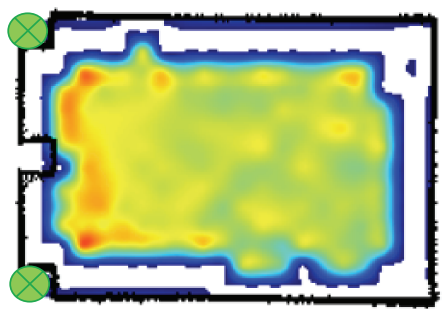

$\operatorname{Max}=27 \mathrm{ppm}$

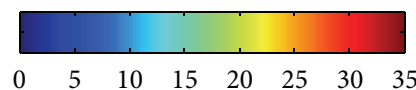

Gas (standard deviation)

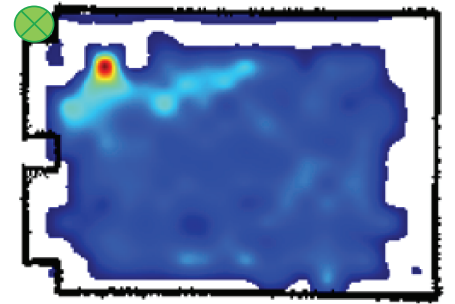

$\operatorname{Max}=17 \mathrm{ppm}$

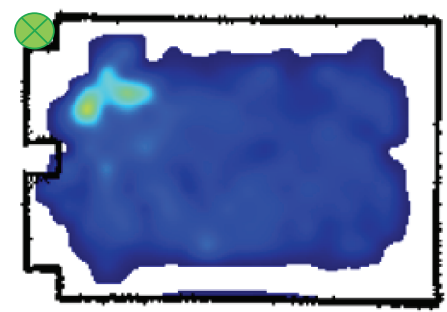

$\operatorname{Max}=9 \mathrm{ppm}$

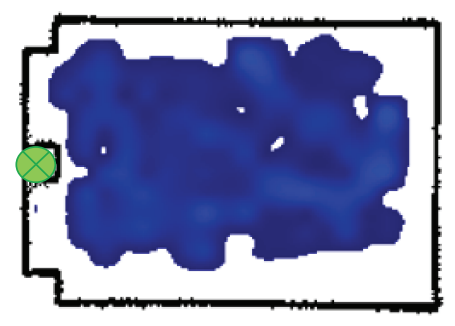

$\operatorname{Max}=3 \mathrm{ppm}$

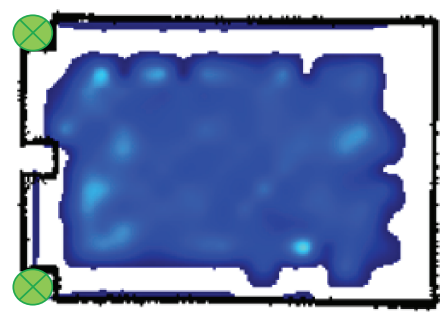

$\operatorname{Max}=6 \mathrm{ppm}$

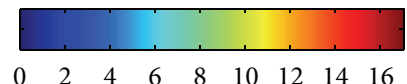

Figure 6: Information layers of the gas concentration in different experimental cases.

Finally, Figure 6, Case 4, had two gas sources located at the corners of the frontal operating simultaneously and the fans were set at $33 \%$ of its maximum power. In this case, the information layer with the values of the maximum peaks measured showed intensity peaks of around $34.5 \mathrm{ppm}$ close to the sources and a double combined diffusion plume with maximum peaks of $14 \mathrm{ppm}$ outside the plumes. The average concentration values had a similar behavior with an average gas intensity value of $27 \mathrm{ppm}$ close to the sources which decreases almost linearly down to $12 \mathrm{ppm}$ up to the opposite side of the wind tunnel. In this case, the information layer showing the standard deviation of the gas concentration measured showed different time-varying peaks close to the walls of the wind tunnel with maximum values of $6 \mathrm{ppm}$ at the peaks and $1.6 \mathrm{ppm}$ outside the areas of the peaks. Therefore, in this case, the expectation is that a mobile robot will be able to locate the gas sources by analyzing the information layers showing the maximum gas concentration measured or the average intensity values although the discrimination will depend on the sensor used to measure the concentrations.

\section{Conclusions}

This paper presents the measurement of the gas concentration and wind intensity inside a turbulent wind tunnel performed with the onboard sensors of a mobile robot in the case of using 
acetone as a gas source target. The alternative grid maps have been summarized as different information layers in order to show the location of the maximum, average, and standard deviation values of the wind intensity and gas concentration. Some previous works such as [13,14] address a similar problem with a mobile robot in the case of having a reduced number of sensor readings per cell. This work proposes the interpretation of multiple sensor readings per cell space in different information layers. The proposed procedure groups and smoothes the data gathered from the sensors in order to create different information layers showing the distribution of the wind and gas concentration profiles inside the turbulent wind tunnel in different operating configurations. Future woks will be focused on using the information maps obtained with the mobile robot for automatic gas source location in real application frameworks.

\section{Conflict of Interests}

The authors declare that there is no conflict of interests regarding the publication of this paper.

\section{Acknowledgments}

This work was partially funded by the Spanish Ministry of Economy and Competitiveness, Plan Nacional de Investigación Científica, Desarrollo e Innovación Tecnológica: TEC2011-26143 and TEC2014-59229-R and by the Government of Catalonia (Comissionat per a Universitats i Recerca, Departament d'Innovació, Universitats i Empresa) and the European Social Fund (ECO/1639/2013).

\section{References}

[1] R. Pfeifer, M. Lungarella, and F. Iida, "Self-organization, embodiment, and biologically inspired robotics," Science, vol. 318, no. 5853, pp. 1088-1093, 2007.

[2] H. Ishida, Y. Wada, and H. Matsukura, "Chemical sensing in robotic applications: a review," IEEE Sensors Journal, vol. 12, no. 11, pp. 3163-3173, 2012.

[3] J. W. Gardner and P. N. Bartlett, "A brief history of electronic noses," Sensors and Actuators B: Chemical, vol. 18, no. 1-3, pp. 210-211, 1994.

[4] J. Zhou, T. Feng, and R. Ye, "Differentiation of eight commercial mushrooms by electronic nose and gas chromatography-mass spectrometry," Journal of Sensors, vol. 2015, Article ID 374013, 14 pages, 2015.

[5] R. A. Russell, "Human-like robot sensing mediated by body heat," Journal of Bionic Engineering, vol. 11, no. 1, pp. 82-89, 2014.

[6] D. Martinez, M. Teixidó, D. Font et al., "Ambient intelligence application based on environmental measurements performed with an assistant mobile robot," Sensors, vol. 14, no. 4, pp. 60456055, 2014.

[7] P. K. Sekhar, J. Zhou, H. Wang, and E. R. Hamblin, "Trace detection of pentaerythritol tetranitrate using electrochemical gas sensors," Journal of Sensors, vol. 2014, Article ID 234607, 6 pages, 2014.

[8] W. Nie, Y. Liu, C. J. Li, and J. Xu, "A gas monitoring and control system in a coal and gas outburst laboratory," Journal of Sensors, vol. 2014, Article ID 172016, 11 pages, 2014.
[9] Y. Wada, M. Trincavelli, Y. Fukazawa, and H. Ishida, "Collecting a database for studying gas distribution mapping and gas source localization with mobile robots," in Proceedings of the International Confederation of Architectural Museums (IACM '10), Shanghai, China, 2010.

[10] S. Thrun, "Robotic mapping: a survey," in Exploring Artificial Intelligence in the New Millennium, pp. 1-35, Morgan Kaufmann Publishers, San Francisco, Calif, USA, 2002.

[11] F.-J. Pei, H.-Y. Li, and Y.-H. Cheng, "An improved FastSLAM system based on distributed structure for autonomous robot navigation," Journal of Sensors, vol. 2014, Article ID 456289, 9 pages, 2014.

[12] E. Guerra, R. Munguia, and A. Grau, "Monocular SLAM for autonomous robots with enhanced features initialization," Sensors, vol. 14, no. 4, pp. 6317-6337, 2014.

[13] A. Lilienthal and T. Duckett, "Building gas concentration gridmaps with a mobile robot," Robotics and Autonomous Systems, vol. 48, no. 1, pp. 3-16, 2004.

[14] A. Loutfi, S. Coradeschi, A. J. Lilienthal, and J. Gonzalez, "Gas distribution mapping of multiple odour sources using a mobile robot," Robotica, vol. 27, no. 2, pp. 311-319, 2009.

[15] V. Hernandez Bennetts, E. Schaffernicht, V. Pomareda, A. J. Lilienthal, S. Marco, and M. Trincavelli, "Combining non selective gas sensors on a mobile robot for identification and mapping of multiple chemical compounds," Sensors, vol. 14, no. 9, pp. 17331-17352, 2014.

[16] M. Turduev, G. Cabrita, M. Kirtay, V. Gazi, and L. Marques, "Experimental studies on chemical concentration map building by a multi-robot system using bio-inspired algorithms," Autonomous Agents and Multi-Agent Systems, vol. 28, no. 1, pp. 72-100, 2014.

[17] P. P. Neumann, S. Asadi, A. J. Lilienthal, M. Bartholmai, and J. H. Schiller, "Autonomous gas-sensitive microdrone: wind vector estimation and gas distribution mapping," IEEE Robotics \& Automation Magazine, vol. 19, no. 1, pp. 50-61, 2012.

[18] J. Fonollosa, I. Rodríguez-Luján, M. Trincavelli, and R. Huerta, "Dataset from chemical gas sensor array in turbulent wind tunnel," Data in Brief, vol. 3, pp. 169-174, 2015.

[19] T. Collins, J. J. Collins, and C. Ryan, "Occupancy grid mapping: an empirical evaluation," in Proceedings of the Mediterranean Conference on Control \& Automation (MED '07), pp. 1-6, Athens, Greece, June 2007.

[20] D. Martínez, J. Moreno, M. Tresanchez et al., "Experimental application of an autonomous mobile robot for gas leak detection in indoor environments," in Proceedings of the 17th International Conference on Information Fusion (FUSION '14), pp. 1-6, IEEE, Salamanca, Spain, July 2014.

[21] J. H. Ahlberg, E. N. Nilson, and J. L. Walsh, The Theory of Splines and Their Applications, Academic Press, New York, NY, USA, 1967.

[22] D. T. Sandwell, "Biharmonic spline interpolation of GEOS-3 and Seasat altimeter data," Geophysical Research Letters, vol. 14, no. 2, pp. 139-142, 1987. 


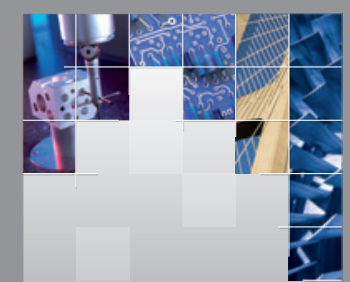

\section{Enfincering}
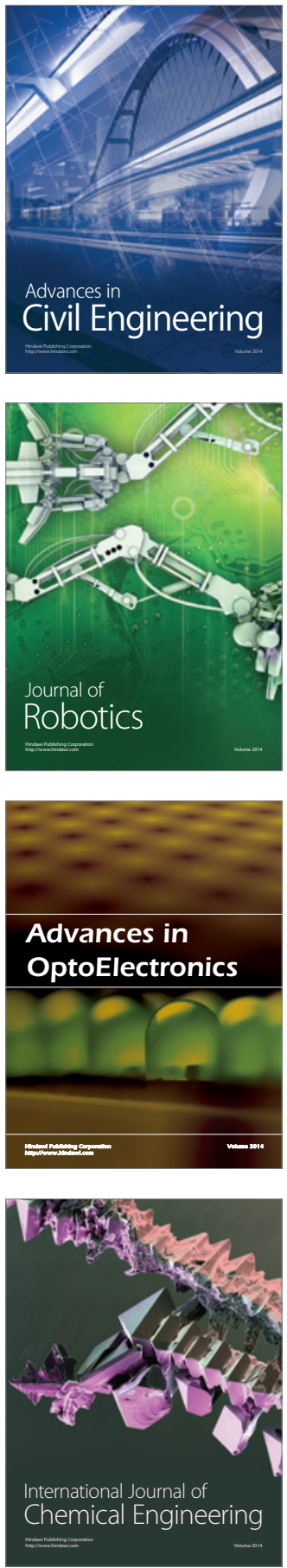

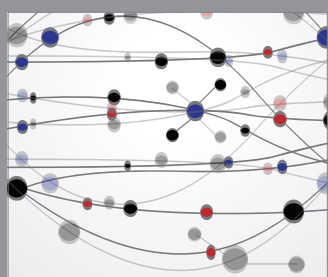

The Scientific World Journal

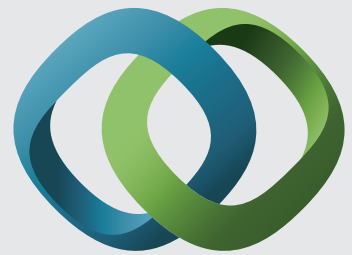

\section{Hindawi}

Submit your manuscripts at

http://www.hindawi.com
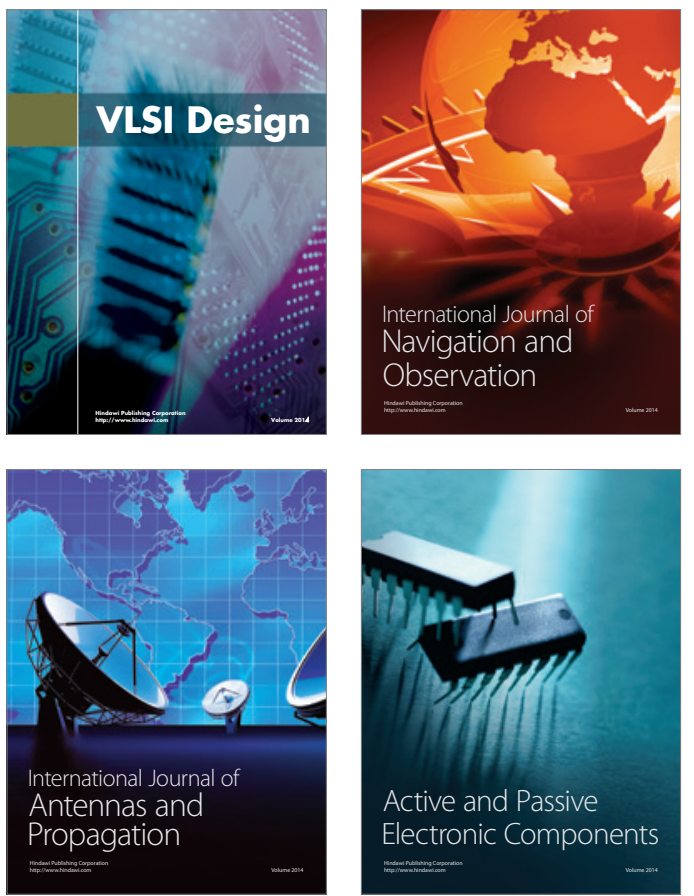
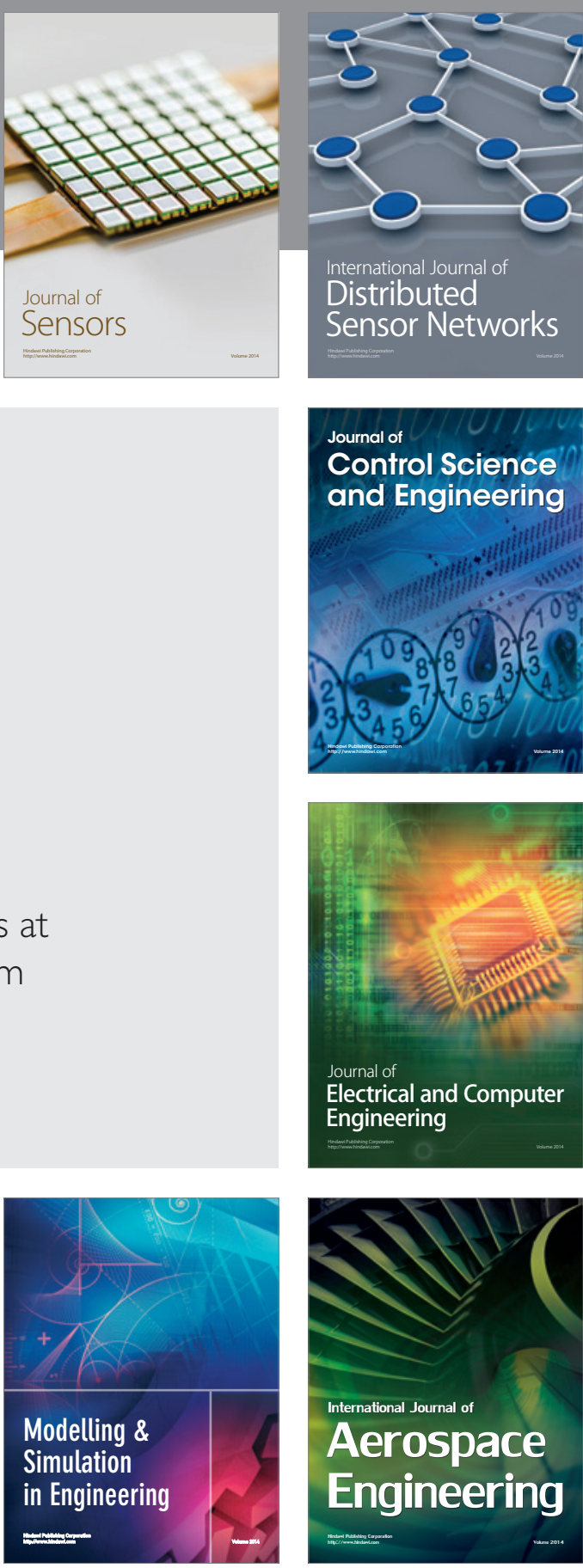

International Journal of

Distributed

Sensor Networks

Journal of

Control Science

and Engineering
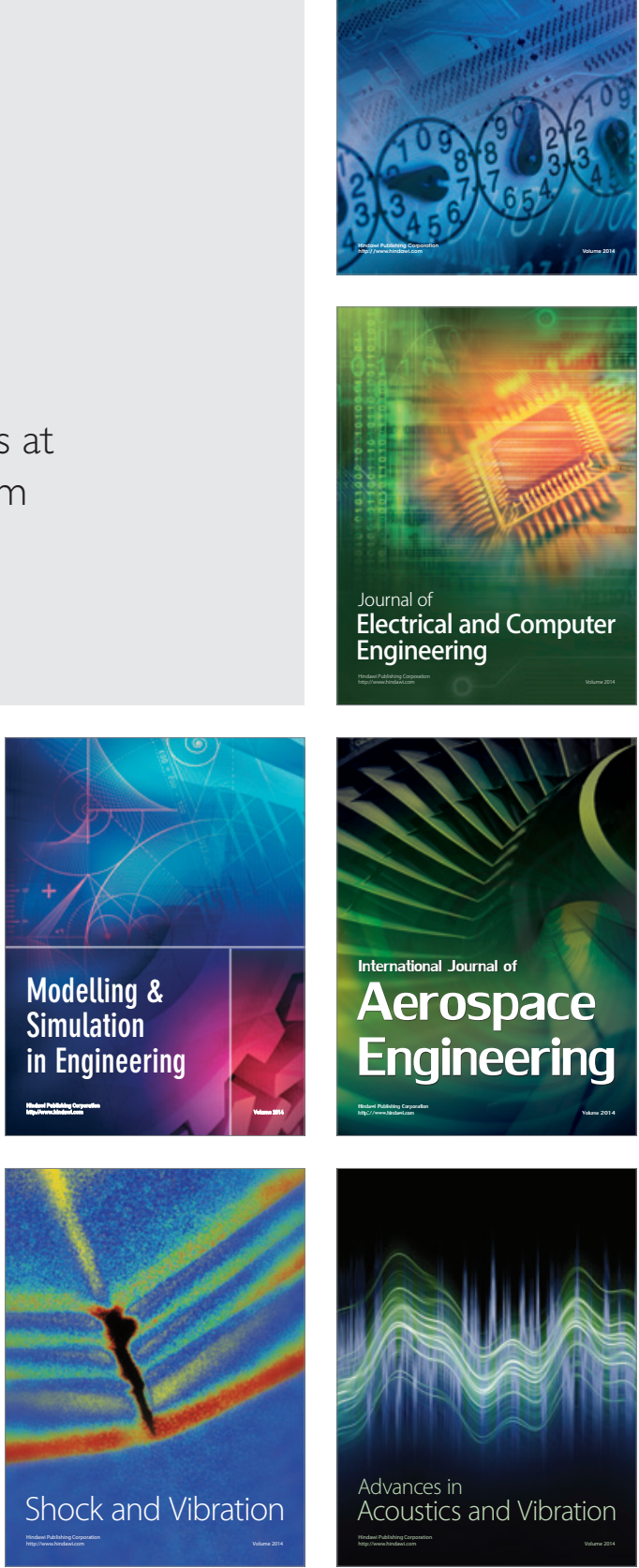